\title{
Harmonized dataset of surface fuels under Alpine, temperate and Mediterranean conditions in Italy. A synthesis supporting fire
}

\section{management}

\author{
Davide Ascoli ${ }^{(1)}$, \\ Giorgio Vacchiano ${ }^{(2)}$, \\ Carla Scarpa ${ }^{(3)}$, \\ Bachisio Arca (3), \\ Anna Barbati (4), \\ Giovanna Battipaglia ${ }^{(5)}$, \\ Mario Elia ${ }^{(6)}$, \\ Assunta Esposito ${ }^{(5)}$, \\ Vittorio Garfi ${ }^{(7)}$, \\ Raffaella Lovreglio ${ }^{(8)}$, \\ Paola Mairota ${ }^{(6)}$, \\ Marco Marchetti ${ }^{(7)}$, \\ Enrico Marchi ${ }^{(9)}$, \\ Simone Meytre ${ }^{(1)}$, \\ Marco Ottaviano ${ }^{(7)}$, \\ Grazia Pellizzaro (3), \\ Rolando Rizzolo ${ }^{(10)}$ \\ Lorenzo Sallustio ${ }^{(7)}$, \\ Michele Salis ${ }^{(3)}$, \\ Costantino Sirca ${ }^{(11-12)}$, \\ Eva Valese ${ }^{(13)}$, \\ Andrea Ventura ${ }^{(3)}$ \\ Valentina Bacciu ${ }^{(12)}$
}

\section{Introduction}

Fire is a widespread disturbance in most vegetation types in Italy under Alpine, temperate and Mediterranean conditions (Elia et al. 2020). Regional fire management

\begin{abstract}
Surface biomass characterization plays a key role in wildfire management. It allows classifying vegetation fuels flammability for fire risk analysis, to define silvicultural prescriptions for fire hazard reduction, to plan prescribed burning, or to model fire behavior and its effects, such as greenhouse gas and pollutant emissions. To facilitate fuel classification and analysis of potential fire behavior and effects in Italy, we harmonized 634 measurements of surface wildland fuels from Alpine, temperate and Mediterranean environments. The dataset provides quantitative data for duff, fine dead fuels and downed woody material, live grasses and shrubs fuel components. Surface fuel data were harmonized by subdividing loads $\left(\mathrm{Mg} \mathrm{ha}^{-1}\right)$ to standard size classes for dead $(0-6$, 6-25 and 25-75 mm) and live $(0-6,6-25 \mathrm{~mm})$ fuels, collecting percent cover and depth/height $(\mathrm{cm})$ of the various fuel components, and classifying observations into 19 fuelbed categories. To ensure comparability with existing vegetation classification systems, we classified each observation according to the European Fuel Map, the Corine Land Cover classes (level IV), the European Forest Types, and the forest categories of the Italian National Forest Inventory. The dataset and a photo description of each fuelbed category are available as Supplementary material. This dataset is the first step to develop several products at the national scale such as: (i) fuel type classification and mapping; (ii) carbon stock and wildfire emission estimates; (iii) calibration of fuel models for the simulation of fire behavior and effects.
\end{abstract}

Keywords: Wildfire, Fire Behavior, Simulation, Fuel Types, Emissions

agencies and National Parks are in an increasing need of fire risk assessment, estimates of fire emissions at different spatial scales, fuel management and prescribed burning planning (Ascoli \& Bovio 2013, Co- rona et al. 2015, Bacciu et al. 2018). The use of decision support systems (DSS) for fire effects modeling, fire behavior simulation and risk analysis is spreading in both the research and operational sectors in Italy

(1) Dept. of Agriculture, Forest and Food Sciences, University of Torino, l.go Paolo Braccini 4, Grugliasco, TO (Italy); (2) Dept. of Agricultural and Environmental Science, University of Milano, v. Celoria 2, Milano (Italy); (3) Institute of Bio-Economy, National Research Council, trav. La Crucca 3, Sassari (Italy); (4) Dept. for Innovation in Biological, Agro-food and Forest systems, University of Tuscia, v. S. Camillo de Lellis snc, VT (Italy); (5) Dept. of Environmental, Biological and Pharmaceutical Sciences and Technologies, University of Campania "Luigi Vanvitelli”, v. Vivaldi 43, 81100 Caserta (Italy); (6) Dept. of Agricultural and Environmental Sciences, University of Bari “A. Moro”, v. Amendola 165/A, 70126 Bari (Italy); (7) Dept. of Biosciences and Territory, University of Molise, c.da Fonte Lappone, Pesche, IS (Italy); (8) Nuoro Forestry School, Dept. of Agriculture, University of Sassari, v. Cristoforo Colombo 1, I-08100 Nuoro (Italy); (9) Dept. of Agriculture, Food, Environment and Forestry, University of Florence, v. S. Bonaventura 13, 50145 Firenze (Italy); (10) Direzione Protezione Civile e Polizia locale, Regione Veneto, Marghera, VE (Italy); (11) University of Sassari, Dip.to di Agraria, Sassari (Italy); (12) Fondazione Centro Euro-Mediterraneo sui Cambiamenti Climatici (CMCC) - IAFES Division, Sassari (Italy); (13) Independent researcher, Agricultural High School "Duca degli Abruzzi”, v. Merlin 1, Padova (Italy)

\section{@ Giorgio Vacchiano (gvacchiano@gmail.com)}

Received: Jul 15, 2020 - Accepted: Oct 26, 2020

Citation: Ascoli D, Vacchiano G, Scarpa C, Arca B, Barbati A, Battipaglia G, Elia M, Esposito A, Garfi V, Lovreglio R, Mairota P, Marchetti M, Marchi E, Meytre S, Ottaviano M, Pellizzaro G, Rizzolo R, Sallustio L, Salis M, Sirca C, Valese E, Ventura A, Bacciu V (2020). Harmonized dataset of surface fuels under Alpine, temperate and Mediterranean conditions in Italy. A synthesis supporting fire management. iForest 13: 513-522. - doi: 10.3832/ifor3587-013 [online 2020-11-13] 
(Salis et al. 2013, Vacchiano et al. 2014, Elia et al. 2015). A quantitative characterization of fuels in Italian forests, shrublands and grasslands is the first step towards developing harmonized fuel datasets to support fire management decisions and calibrating fuel inputs for fire software applications and DSS used by professionals and researchers (Bacciu et al. 2009, Ascoli et al. 2015).

Several approaches have been proposed to quantify and classify fuel complexes, each compatible with a specific modeling system for fire behavior and effects prediction (Sandberg et al. 2001, Dimitrakopoulos 2002, Alvarez et al. 2012, Keane 2013, Cruz et al. 2018). Fire effect models, e.g., FOFEM (Reinhardt et al. 1997), requires several information about fuel components (e.g., litter, downed woody fuel), often based on vegetation types or fuelbed characteristics (Reinhardt et al. 1997, Ottmar et al. 2007). Widespread DSS for fire behavior prediction - such as BehavePlus (Andrews et al. 2005), Farsite (Finney 1998), FlamMap

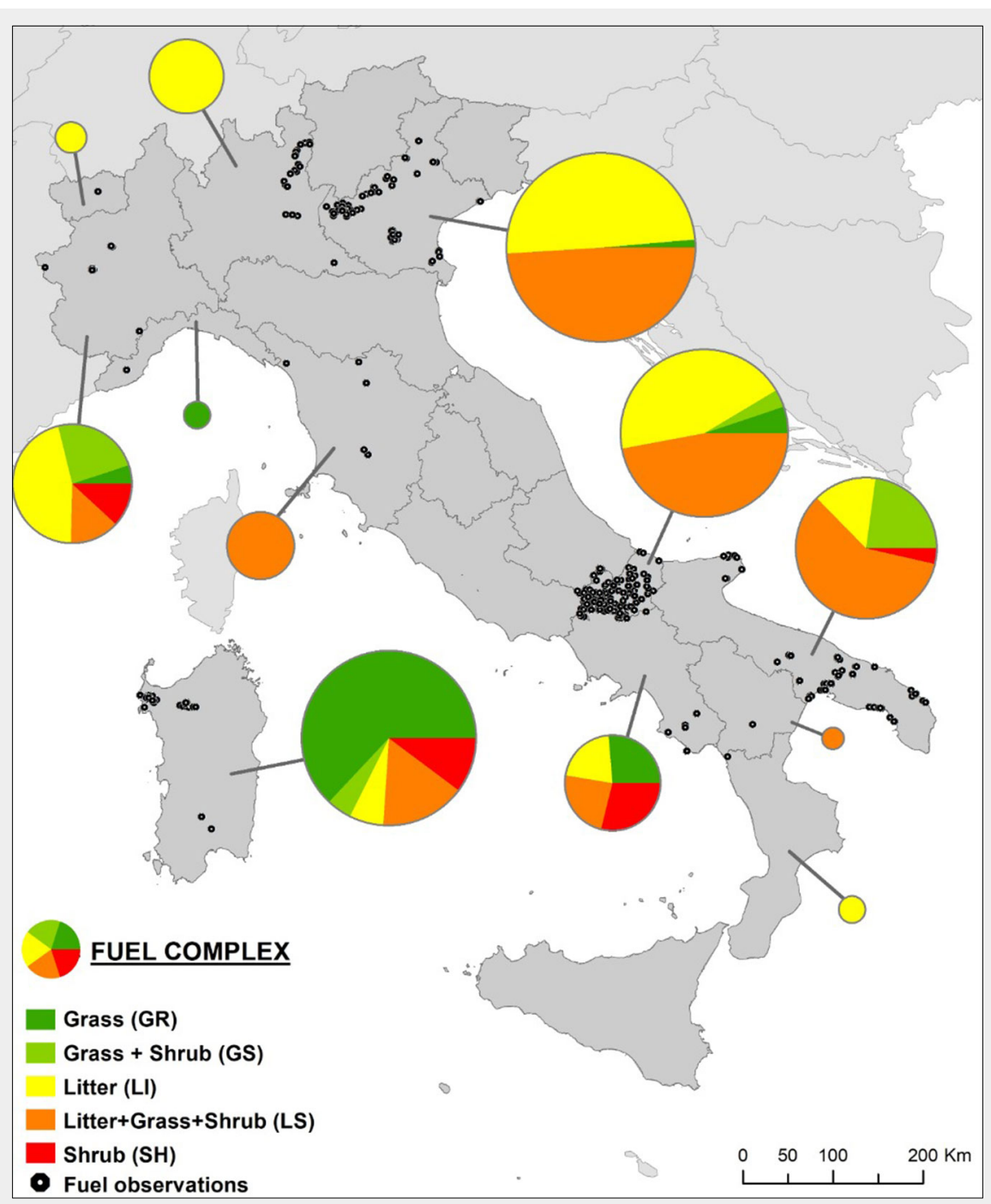

Fig. 1 - Distribution of study sites (black dots) where fuel data were inventoried in Italy. Pie charts represent the relative amount of samples in different fuel complexes (grass only, grass + shrub, litter only, litter + grass + shrub, shrub only). Pie dimension is relative to sample abundance.
(Finney 2006), the Fire and Fuel Extension to the Forest Vegetation Simulator (Andrews 2018), or the Rothermel $R$ package (Vacchiano \& Ascoli 2015) - require a number of parameters represented by "fire behavior fuel models" as input (hereafter "fuel model"), related to surface fuels according to the fuel modeling system developed by Rothermel (1972). However, a source of uncertainty in available fuel description derives from the use of different terminologies to indicate similar fuel classes or categories (Keane 2013). This paper employs the terminology provided by Keane (2015), who defined the fuelbed as the array of biomass types for a given area, further composed by fuel types (e.g., litter, shrubs, grasses, downed woody material and tree crown foliage) and components (e.g., diameter size range; live or dead status) with specific properties. Furthermore, a fuel complex identifies the dominant fuel of a fuelbed (Bebi et al. 2003).

Due to the increased use of Rothermel's based fire software and DSS in Italy by fire managers and researchers (Arca et al. 2007, 2019, Bacciu et al. 2009, Vacchiano \& Ascoli 2015, Ascoli et al. 2015, Elia et al. 2016, Salis et al. 2016), a standardized field protocol for inventorying surface fuels according to Rothermel's fuel models inputs has been proposed in Italy (Bovio \& Ascoli 2013, Bovio et al. 2014). This protocol represented the basis for several unpublished surveys carried out nationwide in the last decade. However, a complete and harmonized inventory of Italian wildland fuels is still lacking.

The aims of the present paper are to: (i) harmonize and make freely available unpublished surface fuel data across major vegetation types in Italy; (ii) categorize data into fuelbeds and establish associations with land cover classes and forest classifications used in Europe and Italy; (iii) summarize fuel data for each fuelbed, providing descriptive statistics of average characteristics and their variability; (iv) release a fuel characteristics dataset useful to calibrate Rothermel's based models and DSS.

\section{Material and methods}

\section{Sampling design}

Provided the spatial distribution of the available data, an a priori sampling design was not applicable to this study, which is instead based on an a posteriori collection of unpublished inventories carried out over the last 10 years. Among existing surveys, only those with data harmonizable in terms of the protocol adopted to characterize the surface fuel complex were included in the study. Most fuel observations in the dataset $(65 \%)$ followed the field protocol described by Bovio \& Ascoli (2013) and Bovio et al. (2014). This uses a mixed approach with both destructive (i.e., vegetation removed and analyzed in the laboratory) and non-destructive techniques (see next paragraph), such as indirect estimates of fuel loads based on allometry equations (Corona et al. 2012, Castagneri et al. 2013, Sirca et al. 2016, Conti et al. 2019). The remaining $35 \%$ of survey data adopted protocols that are similar to the ones mentioned, but applied a fully destructive sampling to the shrub component, as they were mostly carried out in shrubland fuelbeds (Bacciu et al. 2009, Duce et al. 2012, Castagneri et al. 2013, Vacchiano et al. 2014).

The study sites encompass grasslands, shrublands and forest ecosystems growing in Alpine, temperate, and Mediterranean environments throughout a large part of insular and peninsular Italy (Fig. 1). Site selection excluded forests disturbed in the previous 10 years (e.g., affected by wildfire or windthrow, intensively grazed areas, or silvicultural interventions); thus the data set includes 377 sites with a number of randomly located sample plots ranging from 1 to 15 per site (average of $2.28 \pm 2.11$ ). According to the aforementioned protocols (Bovio \& Ascoli 2013, Bovio et al. 2014), the 
structure of the fuel complex was assessed along three 10-m long transects, positioned to form an equilateral triangle (i.e., each transect had a different direction to avoid directional bias). Along each transect, surface litter depth, grasses height, and shrub crown height were measured at $1 \mathrm{~m}$ intervals. Surface litter and duff were collected through destructive sampling in three $0.0625 \mathrm{~m}^{2}$ squares (1 square along each transect); downed wood $<75 \mathrm{~mm}$, grasses and shrub fuels were collected on three 1 $\mathrm{m}^{2}$ squares. Harvested fuels were ovendried in the laboratory at $90{ }^{\circ} \mathrm{C}$ for 12 to 48 hours depending on the fuel component (i.e., 12 hours only for fully cured, very fine grasses) to determine the dry weight (Pellizzaro et al. 2007, Dimitrakopoulos et al. 2010, Ubysz \& Valette 2010). For each sampling point, we calculated the dry weight load of dead (duff, litter, downed woody material, dead fraction of grasses and shrubs) and live (grasses, shrubs) fuel components and structural variables as described in the next paragraph and synthetized in the Fig. S1 (Supplementary material).

\section{Dataset description}

The dataset includes 634 surface fuel samples, each described by 41 variables (Tab. S1), and is available in Excel $^{\circledast}$ (.xlsx) format as Supplementary material (Tab. S3). The first dataset field is an identification code (ID) combining the broad fuel type ( $2^{\text {nd }}$ field), the study site code $\left(3^{\text {rd }}\right.$ field), and the number of the fuel sample collected at each site ( $4^{\text {th }}$ field), useful for filtering data. According to the protocol (Bovio \& Ascoli 2013, Bovio et al. 2014), surveys were carried out during the typical environment-specific fire season, to describe fuel characteristics dynamics (e.g., level of grass curing) in the nature of their flammable status. The field SamplingSeason (Tab. S1) reports winter or summer classes, corresponding to Alpine and Mediterranean fire regime seasons in Italy (Elia et al. 2020), respectively. FuelComplex is a broad fuel classification system to identify the dominant fuel of a fuelbed driving fire spread (i.e., litter, grass, shrubs, grass + shrubs, litter + grass + shrubs). Fields from the $5^{\text {th }}$ position to the $20^{\text {th }}$ are variables that quantitatively characterize each fuel sample (Fig. S1). All fields with a $W$ as the first letter (i.e., Wduff, W1h, W10h, W10oh, WGr, WSh1h, WSh1oh) report fuel load in $\mathrm{Mg}$ ha $^{-1}$ on a dry weight basis. Loads of dead fuels (W1h, W10h, W10oh) are partitioned by particle diameter classes $(<6$ $\mathrm{mm}, 6-25 \mathrm{~mm}, 25-75 \mathrm{~mm},>75 \mathrm{~mm}$ ), corresponding to timelag classes $1 \mathrm{~h}, 10 \mathrm{~h}, 100 \mathrm{~h}$, i.e., the amount of time necessary for dead fuels to lose-gain $63 \%$ of the difference between their initial moisture content and their equilibrium moisture (Brown et al. 1982). Live grasses (WGr) included $<6 \mathrm{~mm}$ fuels only and cured grasses were added to the $1 \mathrm{~h}$ dead fuels. Live shrub fuel load was partitioned in $<6 \mathrm{~mm}$ (WSh1h) and 6-25 mm
(WSh10h) size classes. Fuel cover (\%) and fuelbed depth $(\mathrm{cm})$ of dead fuels (Cdead, Ddead), live grasses (CGr, DGr) and shrubs (CSh, DSh) fuels were measured in $81 \%$ and $79 \%$ of sampling points, respectively. In addition, $45 \%$ of observations included data on duff fuel load (Wduff) - a key component to estimate fire emissions, especially in forest ecosystems - while $96 \%$ of observations reported an estimate of the tree canopy cover (CanCov). Each observation contains information about elevation, slope and aspect, and climate information according to the Köppen-Geiger climate classification (Beck et al. 2018) and the "Worldwide Bioclimatic Classification System" (Rivas-Martínez et al. 2011) available in raster format for Italy at $900 \mathrm{~m}$ grain (Pesaresi et al. 2017). Most sampling sites belong to Köppen-Geiger temperate and mesothermal climates (41\% Csa Mediterranean hot summer, $32 \% \mathrm{Cfb}$ Oceanic, $21 \%$ Cfa Humid subtropical), with a small fraction (6\%) of boreal and tundra climates at the topmost elevations up to $2100 \mathrm{~m}$ a.s.l. From Rivas-Martinez classification, we extracted values for bioclimate, continentality type based on the difference between annual maximum and minimum temperature $(63 \%$ subcontinental and semicontinental, 37\% euoceanic or semihyperoceanic), ombrotype (Tab. S1) based on the annual ombrothermic index (24\% dry and semiarid, $21 \%$ subhumid, $55 \%$ humid and hyperhumid), and thermotype (Tab. S1 in Supplementary material) based on thermicity index and yearly positive temperatures (38\% Thermo-, Meso- and Supramediterranean , 23\% Mesotemperate, 39\% Supra- and Orotemperate).

Fuel observations were then grouped into 19 surface fuelbeds (fields: FuelBed, FuelBedCode - Tab. 1), based on expert opinion considering the vegetation type, dominant fuel, its spatial arrangement and species-specific flammability properties that determine potential surface fire behavior (Ottmar et al. 2007, Keane 2013). For example, fuel observations in Mediterranean conifer forests with a long-needle and porous litter (i.e., Pinus halepensis, P. pinaster and $P$. pinea forests) were grouped into two fuelbeds according to the absence (fuelbed 16) or presence (fuelbed 17) of flammable understory grasses and shrubs. Fuelbeds with codes from 1 to 3 represent grass-dominated fuel complexes, 4 to 6 shrublands, 7 to 12 broadleaved forests, 13 to 17 coniferous forests, while 18 and 19 relate to riparian vegetation and marshes, respectively. A photo description of each fuelbed is provided in Fig. S2 (Supplementary material).

The vegetation physiognomy at each sample point was used to associate (sensu Keane 2013) fuelbed classes to relevant fuel, land cover and forest classification systems. Cross-links were established with existing vegetation classification systems in Europe and in Italy (Tab. 1): (i) European fuel map classification (EFFIS 2017); (ii)
Corine Land Cover, level IV (ISPRA 2010); (iii) European Forest Types (Barbati et al. 2014); and (iv) forest categories of the Italian National Forest Inventory (INFC 2005), for forest fuelbeds only. In some cases, a fuelbed was uniquely associated with a vegetation class. For example, fuelbed 7 "Mountain beech litter", which has a distinctive combustibility due to the compactness of the beech litter and the lack of grasses and shrubs in the understory typical in mountain beech forests (Maringer et al. 2020), was uniquely associated with the "Faggete" class in the INFC (Tab. 1). However, different fuelbeds may be associated to the same vegetation class, and conversely, more vegetation classes may be associated to the same fuelbed description (e.g., fuelbeds 8 and 9 are both associated to the European Forest Type 8.2 and the INFC category "Boschi a rovere, roverella $e$ farnia"), which is a typical issue in fuelbed classification systems (Keane 2013).

In addition to surface fuel data, a bibliographic research on crown foliage and branch fuel loads is presented in Tab. S2 (Supplementary material) with the aim to complement forest fuelbeds (from 7 to 19) with canopy information, necessary for several fire effect models, e.g., FOFEM (Reinhardt et al. 1997).

\section{Results}

Dataset descriptive statistics are presented by the sampled variability across the fuel complexes (i.e., dominant fuel of the fuelbed), and then by the variability within the fuelbeds. A comparison with international established fuel inventories is also presented to verify that the range in loads by fuel components falls within the range of variability in reference inventories (see also the Discussion). Finally, a multivariate analysis was carried out to describe geographical and climatic gradients in the dataset.

\section{Dataset descriptive statistics}

Fuel complexes show distinct characteristics (Fig. 2). Loads of dead fine fuels (size $<6 \mathrm{~mm}$ ) and downed fuels (size 6-75 mm) were generally higher in fuel complexes with litter only (LI), or litter mixed with grasses and shrubs (LS), and shrubs only (SH). Although in grasses the mean load of dead fine fuels was very low, several outliers were recorded (Fig. 2a), mainly due to fuelbed 3 "Continuous tall Mediterranean grasslands", which is dominated by the tall grass Ampelodesmos mauritanicus (Poir.) T. Durand \& Schinz and characterized by relatively high loads of fine dead leaves within the tussock. This represents a species-specific surface fire environment characterizing fire-prone grasslands of the southern Tyrrhenian cost (Incerti et al. 2013) and was therefore identified as a specific fuelbed (fuelbed 3 in Fig. S1). Live fuel load with particle size 6-25 mm was particularly abundant in the shrub fuel complex (Fig. 2d).

Tab. 2 and Tab. 3 provides descriptive sta- 
Tab. 1 - Fuelbed association with the European Forest Fire Information System (EFFIS) fuel map classes, Corine Land Cover classes (level IV), the European Forest Types and the forest categories of the Italian National Forest Inventory (INFC). For fuelbeds 1 to 6,12 and 19, there was no meaningful correspondence ("na") with either the European Forest Types or the forest categories of the Ital ian National Forest Inventory.

\begin{tabular}{|c|c|c|c|c|}
\hline Fuelbed & EFFIS fuel map class & Corine LC IV level & EU Forest Types & INFC Categories \\
\hline $\begin{array}{l}\text { 1. Sparse and very short } \\
\text { grasslands }\end{array}$ & 4. Sparse grasslands & 3212. Discontinuous grassland & na & na \\
\hline 2. Continuous short grasslands & $\begin{array}{l}\text { 5. Mediterranean grasslands } \\
\text { and steppes; } \\
\text { 6. Temperate, Alpine and } \\
\text { Northern grasslands }\end{array}$ & 3211. Continuous grassland & na & na \\
\hline $\begin{array}{l}\text { 3. Continuous tall } \\
\text { Mediterranean grasslands }\end{array}$ & $\begin{array}{l}\text { 5. Mediterranean grasslands } \\
\text { and steppes }\end{array}$ & 3211. Continuous grassland & na & na \\
\hline $\begin{array}{l}\text { 4. Temperate and Alpine } \\
\text { heathlands }\end{array}$ & $\begin{array}{l}\text { 8. Temperate, Alpine and } \\
\text { Northern moors and } \\
\text { heathlands }\end{array}$ & 322. Moors and heathlands & na & na \\
\hline $\begin{array}{l}\text { 5. Short Mediterranean } \\
\text { shrublands and garrigues }\end{array}$ & $\begin{array}{l}\text { 9. Mediterranean open } \\
\text { shrublands (sclerophylous) }\end{array}$ & $\begin{array}{l}\text { 3232. Low maquis and } \\
\text { garrigues }\end{array}$ & na & na \\
\hline $\begin{array}{l}\text { 6. Tall Mediterranean } \\
\text { shrublands and heathlands }\end{array}$ & $\begin{array}{l}\text { 10. Mediterranean shrublands } \\
\text { (sclerophylous) }\end{array}$ & 3231. High maquis & na & na \\
\hline 7. Montane beech litter & $\begin{array}{l}\text { 33. Montane beech forest; } \\
\text { 38. Mixed beech with conifers } \\
\text { forest }\end{array}$ & 3115. Beech forests & $\begin{array}{l}\text { 7.1 South western European } \\
\text { mountainous beech forest; } \\
7.3 \text { Apennine-Corsican } \\
\text { mountainous beech forest }\end{array}$ & Faggete \\
\hline $\begin{array}{l}\text { 8. Compact mesophytic } \\
\text { broadleaved litter }\end{array}$ & $\begin{array}{l}\text { 31. Mesophytic broadleaved } \\
\text { forest }\end{array}$ & $\begin{array}{l}\text { 2241. Young tree plantations } \\
\text { for wood production; } 3112 \text {. } \\
\text { Deciduous oak forests; } 3113 \text {. } \\
\text { Mesophilous broad-leaved } \\
\text { forests; } 3117 \text {. Non-native } \\
\text { broadleaved forests }\end{array}$ & $\begin{array}{l}5.1 \text { Pedunculate oak- } \\
\text { hornbeam forest; } 5.9 \text { Other } \\
\text { mesophytic deciduous forests; } \\
8.2 \text { Turkey oak, Hungarian oak } \\
\text { and Sessile oak forest; } 14 \text { In- } \\
\text { troduced tree species forest }\end{array}$ & $\begin{array}{l}\text { Boschi a rovere, roverella e } \\
\text { farnia; Cerrete, boschi di } \\
\text { farnetto, fragno, vallonea; } \\
\text { Ostrieti, carpineti }\end{array}$ \\
\hline $\begin{array}{l}\text { 9. Porous thermophilous } \\
\text { broadleaved litter }\end{array}$ & $\begin{array}{l}\text { 30. Thermophilous } \\
\text { broadleaved forest }\end{array}$ & $\begin{array}{l}\text { 3112. Deciduous oak forests } \\
\text { 3113. Mesophilous broad- } \\
\text { leaved forests }\end{array}$ & $\begin{array}{l}8.1 \text { Downy oak forest; } 8.2 \\
\text { Turkey oak, Hungarian oak and } \\
\text { Sessile oak forest; } 8.8 \text { Other } \\
\text { thermophilous deciduous } \\
\text { forests }\end{array}$ & $\begin{array}{l}\text { Boschi a rovere, roverella e } \\
\text { farnia; Cerrete, boschi di } \\
\text { farnetto, fragno, vallonea; } \\
\text { Ostrieti, carpineti }\end{array}$ \\
\hline $\begin{array}{l}\text { 10. Mediterranean evergreen } \\
\text { broadleaved litter }\end{array}$ & $\begin{array}{l}\text { 29. Mediterranean evergreen } \\
\text { broadleaved forest }\end{array}$ & $\begin{array}{l}\text { 3111. Mediterranean } \\
\text { evergreen oak forests }\end{array}$ & $\begin{array}{l}\text { 9.1 Mediterranean evergreen } \\
\text { oak forest }\end{array}$ & $\begin{array}{l}\text { Leccete; Sugherete; Altre } \\
\text { latifoglie sempreverdi }\end{array}$ \\
\hline 11. Long broadleaved litter & $\begin{array}{l}\text { 30. Thermophilous } \\
\text { broadleaved forest }\end{array}$ & 3114. Chestnut forests & 8.7 Chestnut forest & Castagneti \\
\hline 12. Eucaliptus litter & na & $\begin{array}{l}\text { 3117. Non-native broad-leaved } \\
\text { forests }\end{array}$ & $\begin{array}{l}14.2 \text { Plantations of not-site- } \\
\text { native species and self-sown } \\
\text { exotic forest }\end{array}$ & na \\
\hline $\begin{array}{l}\text { 13. Alpine and Mediterranean } \\
\text { short needled conifer litter }\end{array}$ & $\begin{array}{l}\text { 23. Mediterranean montane } \\
\text { short needled conifer forest } \\
\text { (fir); } \\
\text { 26. Alpine short needled } \\
\text { conifer forest (fir, alpine } \\
\text { spruce) }\end{array}$ & $\begin{array}{l}\text { 3123. Silver fir and/or Norway } \\
\text { spruce forests }\end{array}$ & $\begin{array}{l}\text { 3.2 Subalpine and } \\
\text { mountainous spruce and } \\
\text { mountainous mixed spruce- } \\
\text { silver fir forest; } 7.9 \\
\text { Mountainous Silver fir forest }\end{array}$ & $\begin{array}{l}\text { Boschi di abete rosso; Boschi } \\
\text { di abete bianco }\end{array}$ \\
\hline $\begin{array}{l}\text { 14. Montane long needled } \\
\text { conifer litter }\end{array}$ & $\begin{array}{l}\text { 22. Mediterranean montane } \\
\text { long needled conifer forest } \\
\text { (Black and scots pines); } \\
\text { 25. Alpine long needled } \\
\text { conifer forest (pine) }\end{array}$ & $\begin{array}{l}\text { 3122. Mountain and oro- } \\
\text { Mediterranean pine forests; } \\
3124 \text {. Larch and/or Arolla pine } \\
\text { forests; } \\
3125 \text {. Non-native coniferous } \\
\text { forests }\end{array}$ & $\begin{array}{l}\text { 10.2 Mediterranean and } \\
\text { Anatolian Black pine forest; } \\
\text { 14.2 Plantations of not-site- } \\
\text { native species and self-sown } \\
\text { exotic forest; } 3.1 \text { Subalpine } \\
\text { larch-arolla pine and dwarf } \\
\text { pine forest; } 3.3 \text { Alpine Scots } \\
\text { pine and Black pine }\end{array}$ & $\begin{array}{l}\text { Pinete di pino silvestre e } \\
\text { montano; Pinete di pino nero, } \\
\text { laricio e loricato; Piantagioni } \\
\text { di conifere }\end{array}$ \\
\hline $\begin{array}{l}\text { 15. Montane long needled } \\
\text { conifer understory with shrubs }\end{array}$ & $\begin{array}{l}\text { 22. Mediterranean montane } \\
\text { long needled conifer forest } \\
\text { (Black and scots pines); } \\
\text { 25. Alpine long needled } \\
\text { conifer forest (pine) }\end{array}$ & $\begin{array}{l}\text { 3122. Mountain and oro- } \\
\text { Mediterranean pine forests } \\
\text { 3124. Larch and/or Arolla pine } \\
\text { forests }\end{array}$ & $\begin{array}{l}\text { 10.2 Mediterranean and } \\
\text { Anatolian Black pine forest; } \\
\text { 14.2 Plantations of not-site- } \\
\text { native species and self-sown } \\
\text { exotic forest; } 3.1 \text { Subalpine } \\
\text { larch-arolla pine and dwarf } \\
\text { pine forest; } 3.3 \text { Alpine Scots } \\
\text { pine and Black pine }\end{array}$ & $\begin{array}{l}\text { Pinete di pino silvestre e } \\
\text { montano; Pinete di pino nero, } \\
\text { laricio e loricato; Piantagioni } \\
\text { di conifere }\end{array}$ \\
\hline $\begin{array}{l}\text { 16. Mediterranean long } \\
\text { needled conifer litter }\end{array}$ & $\begin{array}{l}\text { 20. Mediterranean long } \\
\text { needled conifer forest (Med. } \\
\text { pines) }\end{array}$ & $\begin{array}{l}\text { 3121. Mediterranean pine } \\
\text { forests }\end{array}$ & $\begin{array}{l}10.1 \text { Mediterranean pine } \\
\text { forest }\end{array}$ & Pinete di pini mediterrane \\
\hline $\begin{array}{l}\text { 17. Mediterranean long } \\
\text { needled conifer understory } \\
\text { with shrubs }\end{array}$ & $\begin{array}{l}\text { 20. Mediterranean long } \\
\text { needled conifer forest (Med. } \\
\text { pines) }\end{array}$ & $\begin{array}{l}\text { 3121. Mediterranean pine } \\
\text { forests }\end{array}$ & $\begin{array}{l}10.1 \text { Mediterranean pine } \\
\text { forest }\end{array}$ & Pinete di pini mediterrane \\
\hline 18. Riparian vegetation & 39. Riparian vegetation & 3116. Hygrophilous forests & 12.1 Riparian forest & Boschi igrofili \\
\hline 19. Aquatic marshes & 41. Aquatic marshes & na & na & na \\
\hline
\end{tabular}


Fig. 2 - Boxplot of fuel load (Mg ha-1) by fuel complex $(\mathrm{GR}=$ grass; $\mathrm{GS}=$ grass + shrub; LI = litter only; LS = litter + grass + shrub; SH = shrub only) and fuel com ponents: (a) Dead fuel with particle size < $6 \mathrm{~mm}$; (b) larger dead downed fuels (size 6-75 mm); (c) live grass fuels $(<6 \mathrm{~mm})$; (d) live shrub fuels ( $<25 \mathrm{~mm})$. Data excluded: duff fuel component; fuelbed 19 "Aquatic marshes".

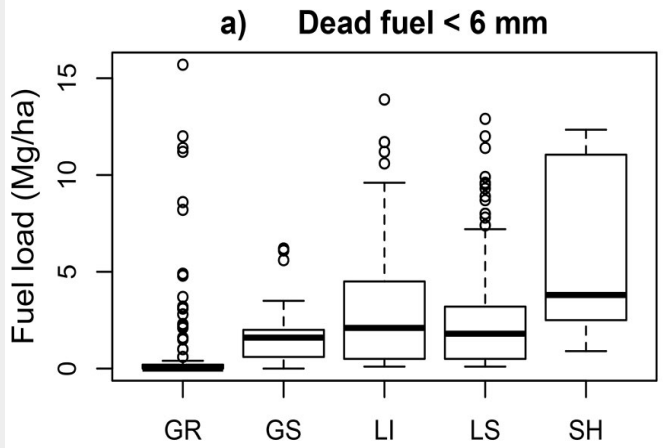

c) Live grass $<6 \mathrm{~mm}$

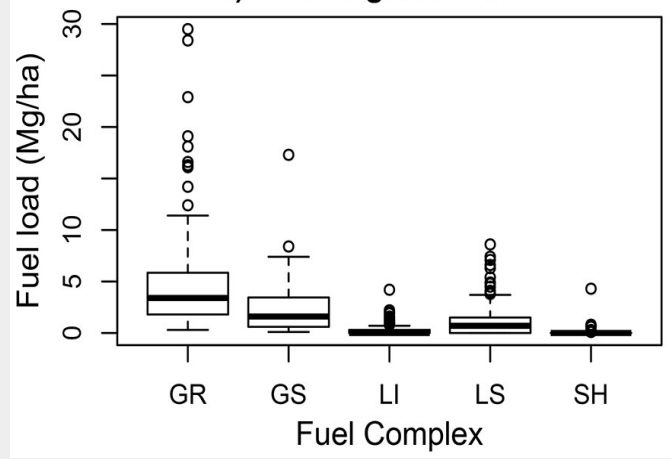

b) Dead downed fuel $6-75 \mathrm{~mm}$

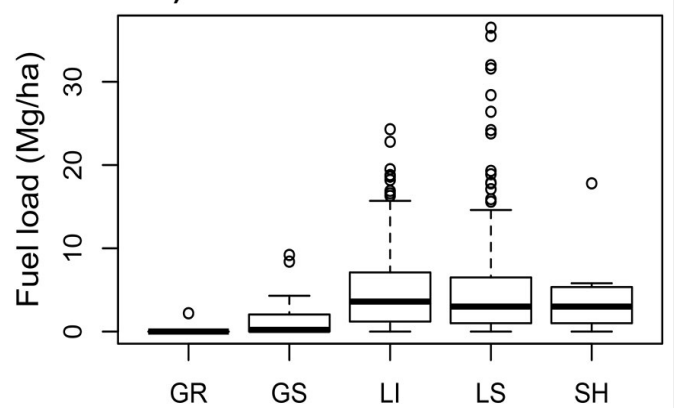

d) Live shrub $<25 \mathrm{~mm}$

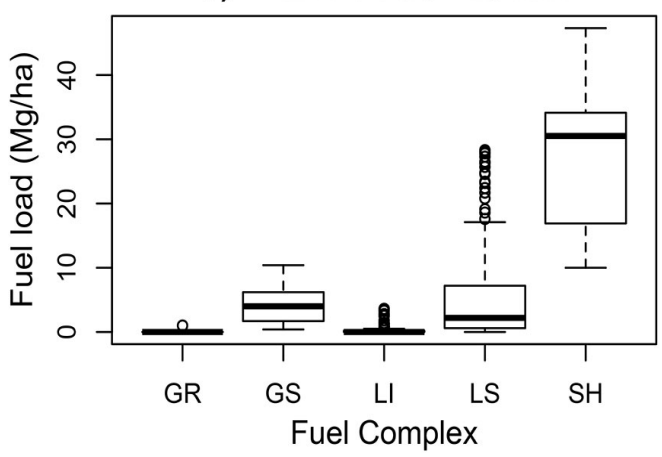

tistics of mean characteristics and variabil- aquatic fuelbeds (Tab. 3). The fuelbed with $2.47 \mathrm{Mg}$ ha-1. Fuelbed 19 "Aquatic marshes" ity of fuel loads and cover for grasslands the lowest value in total surface fuel load shows the highest total surface fuel load and shrublands fuelbeds (Tab. 2) and for (excluding duff) is fuelbed 1 "Sparse and with an average of $80 \mathrm{Mg}^{-1}$. Although broadleaved and coniferous forests, and very short grasslands" with an average of sharing the same vegetation classes (Tab.

Tab. 2 - Number of observations (Count), mean fuel load (Mg ha-1), standard deviation (STD), range (min-max) and interquartile range (IQR) by vegetation type (grassland, shrubland), fuelbed and fuel component. Variable names: Duff: fuel duff load; Dead 1h: dead fuels with particle size $<6 \mathrm{~mm}$; Dead (10h, 10oh): dead fuels with particle size 6-25 mm, 25-75 mm; Live fuels (grass, shrub): live grasses $<6 \mathrm{~mm}$ and live shrubs $<25 \mathrm{~mm}$; Total load: total fuel load (dead + live fuels, but duff excluded).

\begin{tabular}{|c|c|c|c|c|c|c|c|}
\hline Type & Fuelbed & Fuel component & Count & Mean & STD & Range & IQR \\
\hline \multirow{14}{*}{$\begin{array}{l}\tilde{y} \\
\frac{n}{\tilde{T}} \\
\overline{\tilde{n}} \\
\tilde{y} \\
\frac{0}{0}\end{array}$} & \multirow{5}{*}{$\begin{array}{l}\text { 1. Sparse and very short } \\
\text { grasslands }\end{array}$} & Duff & na & na & na & na & na \\
\hline & & Dead 1h & 41 & 0.08 & 0.31 & $0-1.7$ & 0 \\
\hline & & Dead (10h, 100h) & 41 & 0.08 & 0.37 & $0-2.2$ & 0 \\
\hline & & Live fuels (grass, shrub) & 41 & 2.32 & 1.48 & $0.3-7.2$ & 1.4 \\
\hline & & Total load & 41 & 2.47 & 1.66 & $0.3-8.9$ & 1.5 \\
\hline & \multirow{4}{*}{$\begin{array}{l}\text { 2. Continuous short } \\
\text { grasslands }\end{array}$} & Duff & 4 & 23.7 & 24.78 & $4.6-60.1$ & 15.6 \\
\hline & & Dead 1h & 43 & 0.28 & 0.9 & $0-4.8$ & 0 \\
\hline & & Live fuels (grass, shrub) & 43 & 3.77 & 2.03 & $0.5-10.3$ & 2.6 \\
\hline & & Total load & 43 & 4.08 & 2.27 & $0.5-10.8$ & 3 \\
\hline & \multirow{5}{*}{$\begin{array}{l}\text { 3. Continuous tall } \\
\text { Mediterranean grasslands }\end{array}$} & Duff & 25 & na & na & na & na \\
\hline & & Dead $1 \mathrm{~h}$ & 25 & 3.96 & 4.54 & $0-15.7$ & 4.3 \\
\hline & & Dead (10h, 100h) & 25 & 0 & 0 & $0-0$ & 0 \\
\hline & & Live fuels (grass, shrub) & 25 & 12.26 & 7.25 & $3.5-29.5$ & 7.8 \\
\hline & & Total load & 25 & 16.17 & 10.44 & $3.5-40.7$ & 10.2 \\
\hline \multirow{14}{*}{$\begin{array}{l}\frac{n}{0} \\
\frac{c}{0} \\
\frac{0}{0} \\
0 \\
\frac{1}{2} \\
\text { 心 }\end{array}$} & \multirow{4}{*}{$\begin{array}{l}\text { 4. Temperate and Alpine } \\
\text { heathlands }\end{array}$} & Dead $1 \mathrm{~h}$ & 11 & 2.35 & 2.38 & $0.03-6.2$ & 3 \\
\hline & & Dead (10h, 100h) & 11 & 0 & 0 & $0-0$ & 0 \\
\hline & & Live fuels (grass, shrub) & 11 & 6.15 & 2.37 & $3.3-10.6$ & 3.4 \\
\hline & & Total load & 11 & 8.5 & 4.33 & $3.6-16.2$ & 5.6 \\
\hline & \multirow{5}{*}{$\begin{array}{l}\text { 5. Short Mediterranean } \\
\text { shrublands and garrigues }\end{array}$} & Duff & 25 & 6.89 & 4.36 & $0.2-16.7$ & 5.5 \\
\hline & & Dead 1h & 51 & 2.26 & 2.19 & $0-12.9$ & 1.9 \\
\hline & & Dead (10h, 100h) & 51 & 2.92 & 5.5 & $0-32$ & 2.7 \\
\hline & & Live fuels (grass, shrub) & 51 & 10.35 & 7.69 & $1.8-37.5$ & 6.6 \\
\hline & & Total load & 51 & 15.52 & 11.03 & $2.5-54.5$ & 10.5 \\
\hline & \multirow{5}{*}{$\begin{array}{l}\text { 6. Tall Mediterranean } \\
\text { shrublands and heathlands }\end{array}$} & Duff & 11 & 11.65 & 4.24 & $6.5-20.1$ & 5.4 \\
\hline & & Dead $1 \mathrm{~h}$ & 39 & 5.33 & 4.34 & $0.7-12.3$ & 8.4 \\
\hline & & Dead (10h, 100h) & 39 & 3.55 & 3.2 & $0-17.8$ & 3.8 \\
\hline & & Live fuels (grass, shrub) & 39 & 25.53 & 8.67 & $10.4-47.3$ & 15.7 \\
\hline & & Total load & 39 & 34.42 & 12.59 & $16.8-53.3$ & 25.3 \\
\hline
\end{tabular}


Tab. 3 - Number of observations (Count) and fuel load $\left(\mathrm{Mg} \mathrm{ha}^{-1}\right)$ by vegetation type (broadleaved forests, conifer forests, aquatic vegetation), fuelbed and fuel component. Mean, standard deviation (STD), range (min-max) and interquartile range (IQR) are reported. Variable names: Duff: duff fuel load; Dead 1h: dead fuels with particle size $<6 \mathrm{~mm}$; Dead (10h, 10oh): dead fuels with particle size 6-25 mm and 25-75 mm, respectively; Live fuels (grass, shrub): live grasses $<6 \mathrm{~mm}$ and live shrubs $<25 \mathrm{~mm}$; Total load: total fuel load (dead + live fuels, excluding duff).

\begin{tabular}{|c|c|c|c|c|c|c|c|}
\hline Type & Fuelbeds & Fuel component & Count & Mean & STD & Range & IQR \\
\hline \multirow{27}{*}{ 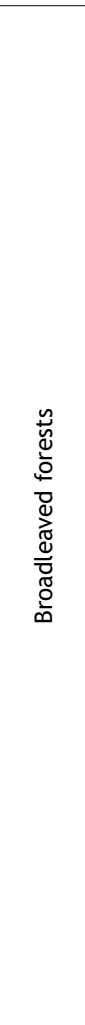 } & \multirow[t]{5}{*}{ 7. Montane beech litter } & Duff & 7 & 35.06 & 26.75 & $9.3-90.1$ & 16.5 \\
\hline & & Dead $1 \mathrm{~h}$ & 17 & 1.88 & 2.37 & $0.1-6.74$ & 3.1 \\
\hline & & Dead (10h, 100h) & 17 & 4.09 & 3.91 & $0.3-14.8$ & 3.3 \\
\hline & & Live fuels (grass, shrub) & 17 & 0.24 & 0.55 & $0-2.2$ & 0.2 \\
\hline & & Total load & 17 & 6.2 & 4.26 & $0.5-14.9$ & 7.1 \\
\hline & \multirow{4}{*}{$\begin{array}{l}\text { 8. Compact mesophitic } \\
\text { broadleaved litter }\end{array}$} & Duff & 68 & 33.07 & 25.84 & $2.3-92.4$ & 40.1 \\
\hline & & Dead $1 \mathrm{~h}$ & 95 & 2.18 & 1.87 & $0.1-8$ & 3.1 \\
\hline & & Live fuels (grass, shrub) & 95 & 1.61 & 2.54 & $0-16.5$ & 1.9 \\
\hline & & Total load & 95 & 10.63 & 7.14 & $0.6-37.6$ & 9.4 \\
\hline & \multirow{5}{*}{$\begin{array}{l}\text { 9. Porous thermophilous } \\
\text { broadleaved litter }\end{array}$} & Duff & 9 & 39.87 & 31.65 & $3-97$ & 36.6 \\
\hline & & Dead 1h & 66 & 1.73 & 2.35 & $0.1-10.6$ & 3 \\
\hline & & Dead (10h, 100h) & 66 & 3.01 & 3.33 & $0-13.3$ & 4.2 \\
\hline & & Live fuels (grass, shrub) & 66 & 1.58 & 1.83 & $0-9.8$ & 2.1 \\
\hline & & Total load & 66 & 6.32 & 3.84 & $1-21$ & 4.4 \\
\hline & \multirow{3}{*}{$\begin{array}{l}\text { 10. Mediterranean } \\
\text { evergreen broadleaved litter }\end{array}$} & Dead (10h, 100h) & 19 & 4.69 & 4.82 & $0.2-15.9$ & 7.1 \\
\hline & & Live fuels (grass, shrub) & 19 & 3.76 & 6.52 & $0-27.3$ & 2.7 \\
\hline & & Total load & 19 & 10.71 & 7.74 & $2.3-31.7$ & 9.5 \\
\hline & \multirow[t]{5}{*}{ 11. Long broadleaved litter } & Duff & 33 & 35.69 & 27.5 & $5.8-104$ & 41.5 \\
\hline & & Dead $1 \mathrm{~h}$ & 46 & 2.52 & 2.65 & $0.1-11.4$ & 1.9 \\
\hline & & Dead (10h, 100h) & 46 & 6.47 & 4.84 & $0.7-17.1$ & 7.3 \\
\hline & & Live fuels (grass, shrub) & 46 & 2.08 & 2.83 & $0-13.7$ & 2 \\
\hline & & Total load & 46 & 11.06 & 6.05 & $2-22.5$ & 10.6 \\
\hline & \multirow[t]{5}{*}{ 12. Eucaliptus litter } & Duff & na & na & na & na & na \\
\hline & & Dead 1h & 9 & 9.08 & 4 & $0.1-13.9$ & 4.2 \\
\hline & & Dead (10h, 100h) & 9 & 4.41 & 1.39 & $2.5-6.4$ & 1.8 \\
\hline & & Live fuels (grass, shrub) & 9 & 0.26 & 0.31 & $0-0.9$ & 0.4 \\
\hline & & Total load & 9 & 13.74 & 4.63 & $4.6-20.7$ & 4 \\
\hline \multirow{23}{*}{ 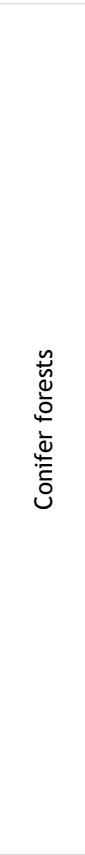 } & \multirow{3}{*}{$\begin{array}{l}\text { 13. Alpine and } \\
\text { Mediterranean short needled } \\
\text { conifer litter }\end{array}$} & Duff & 10 & 39.36 & 16.73 & $19.8-63.8$ & 25.6 \\
\hline & & Dead $1 \mathrm{~h}$ & 15 & 2.36 & 2.02 & $0.1-5.9$ & 3.8 \\
\hline & & Total load & 15 & 6.91 & 2.29 & $3-11.7$ & 2.9 \\
\hline & \multirow{5}{*}{$\begin{array}{l}\text { 14. Montane long needled } \\
\text { conifer litter }\end{array}$} & Duff & 13 & 39.58 & 30.17 & $1.1-93.1$ & 49.9 \\
\hline & & Dead 1h & 36 & 3.32 & 2.63 & $0.1-8.7$ & 4.2 \\
\hline & & Dead (10h, 100h) & 36 & 3.96 & 3.75 & $0-14.4$ & 4.9 \\
\hline & & Live fuels (grass, shrub) & 36 & 0.79 & 1.02 & $0-4.7$ & 0.8 \\
\hline & & Total load & 36 & 8.07 & 5.29 & $0.7-22.9$ & 7.5 \\
\hline & \multirow{5}{*}{$\begin{array}{l}\text { 15. Montane long needled } \\
\text { conifer understory with } \\
\text { shrubs }\end{array}$} & Duff & 24 & 23.92 & 20.1 & $2.7-74.1$ & 11.7 \\
\hline & & Dead $1 \mathrm{~h}$ & 24 & 1.11 & 0.94 & $0.1-3.2$ & 0.8 \\
\hline & & Dead (10h, 100h) & 24 & 11.61 & 11.88 & $0-36.5$ & 17.2 \\
\hline & & Live fuels (grass, shrub) & 24 & 4.5 & 3.26 & $0.5-12.9$ & 3.8 \\
\hline & & Total load & 24 & 17.23 & 12.96 & $2.7-45.3$ & 19.9 \\
\hline & \multirow{5}{*}{$\begin{array}{l}\text { 16. Mediterranean long } \\
\text { needled conifer litter }\end{array}$} & Duff & 12 & 31.81 & 25.73 & $3.4-96.6$ & 25.6 \\
\hline & & Dead 1h & 29 & 2.43 & 1.52 & $0.1-5.6$ & 2.2 \\
\hline & & Dead (10h, 100h) & 29 & 3.3 & 3.78 & $0.2-16.9$ & 2.6 \\
\hline & & Live fuels (grass, shrub) & 29 & 0.89 & 0.99 & $0-4.3$ & 1.1 \\
\hline & & Total load & 29 & 6.61 & 4.23 & $0.8-19.7$ & 2.9 \\
\hline & & Duff & 18 & 32.83 & 25.06 & $22.7-91.4$ & 18.9 \\
\hline & needled conifer understory & Dead $1 \mathrm{~h}$ & 44 & 4.31 & 2.77 & $0.5-12$ & 3.1 \\
\hline & & Dead (10h, 100h) & 44 & 3.42 & 3.28 & $0-14.2$ & 3.7 \\
\hline & & Live fuels (grass, shrub) & 44 & 11.3 & 8.78 & $1.1-29.3$ & 11.9 \\
\hline & & Total load & 44 & 20.13 & 11.11 & $4.7-42$ & 16.4 \\
\hline & 18. Riparian vegetation & Duff & na & na & na & na & na \\
\hline & & Dead 1h & - & 0.49 & 0.54 & $0.1-1.2$ & 0.9 \\
\hline 으 & & Dead (10h, 100h) & 8 & 3.6 & 4.2 & $0.7-10.7$ & 3.7 \\
\hline 苞 & & Live fuels (grass, shrub) & 8 & 3.11 & 4.04 & $0.9-13$ & 0.8 \\
\hline ळ్ & & Total load & 8 & 7.2 & 4.94 & $2.6-14.6$ & 8.5 \\
\hline u & 19. Aquatic & Duff & na & na & na & na & na \\
\hline 竞 & marshes & Dead $1 \mathrm{~h}$ & 10 & 15.22 & 5.43 & $9-26.5$ & 7.2 \\
\hline 守 & & Dead (10h, 100h) & 10 & 17.73 & 5.54 & $8.1-25.1$ & 7.9 \\
\hline & & Live fuels (grass, shrub) & 10 & 47.1 & 20.58 & $24.7-76$ & 36.8 \\
\hline & & Total load & 10 & 80.05 & 20.69 & $53.4-112.7$ & 32.4 \\
\hline
\end{tabular}


), long-needle conifer forests of both montane and Mediterranean areas showed marked differences between fuelbed with litter fuels only (fuelbeds 14 and 16) and litter mixed with grasses and shrubs (fuelbeds 15 and 17). For example, shrub live loads (particle size <25 mm), cover and depth in fuelbeds 16 and 17 was 0.87 vs. $11.30 \mathrm{Mg} \mathrm{ha}^{-1}, 12 \%$ vs. $45 \%$, and $47 \mathrm{~cm}$ vs. 157 $\mathrm{cm}$, respectively, highlighting the marked difference among these fuelbeds, though associations to vegetation classifications are the same (Tab. 3).

\section{Comparison with international dataset}

To verify the congruence of fuel load values devised from the present study with those of similar fuelbeds, the fuel dataset was compared to the Fuel Characteristics Classification System (FCCS - Ottmar et al. 2007). The FCCS stores and classifies fuels data as fuelbeds across the United States, and provides a description of fuel characteristics and properties for 6 horizontal strata including the duff, litter, downed woody material, grasses, and tree canopy, obtained from literature and field data sets. It represents a helpful way for cataloging fuelbeds consumed during the fire (Ottmar et al. 2007, Ottmar 2014). Although the FCCS has been created for the United States, it can be used as reference for different ecosystems, thanks to its accurate description of the horizontal strata of each fuel. When comparing the Italian fuel dataset and FCCS for both total fine fuels (size $<6 \mathrm{~mm}$ ), larger fuels (size 6-75 $\mathrm{mm}$ ) and duff fuels (Fig. 3), fuel loads in each size class did not differ markedly between the two (except for a higher load of fine fuels in F(CS), which suggests that the Italian inventory explores the fuel data space in a fairly complete way.

\section{Multivariate relationship in the dataset}

To test for relationships between fuel loads and topographical and climatic drivers, we carried out a Redundancy Analysis (RDA). Response variables were $1 \mathrm{~h}, 10 \mathrm{~h}$ and $100 \mathrm{~h}$ dead fuel loads, live grasses and live shrubs fuel loads; predictors were elevation, slope, southness (calculated as negative cosine of aspect), canopy cover, ombrotype, thermotype and continentality type according to Pesaresi et al. (2017). Observations were scaled to unit variance; the model was chosen by forward model selection based on adjusted $\mathrm{R}^{2}$ using the "ordiR2step" function in the "vegan" package for R (R Core Team 2019). The final model was checked for collinearity by making sure that variance Inflation factors for each predictors were $<10$. The significance of the model, predictors, and RDA axes was checked by an ANOVA-like permutation test with 999 iterations ( $a=0.05$ ).

The final model had an adjusted $\mathrm{R}^{2}$ of 0.16 . The first four RDA axes were significant, and explained $62.3 \%, 25.8 \%, 5.3 \%$ and $3.5 \%$ of the model variance, respectively. Axis 1 was strongly correlated to canopy

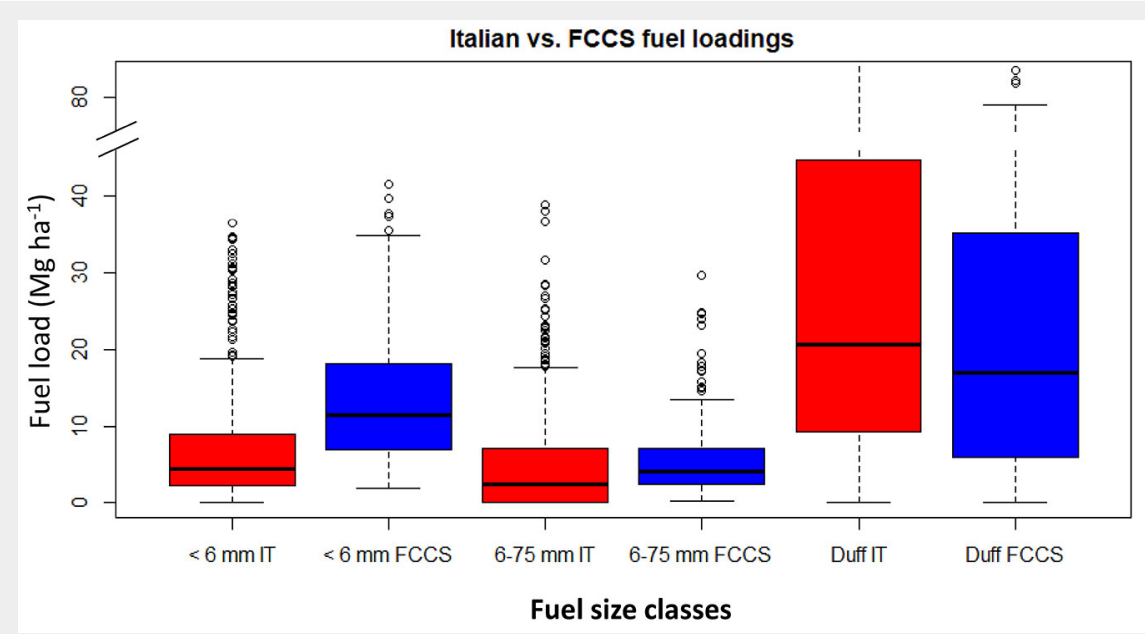

Fig. 3 - Comparison between total load $\left(\mathrm{Mg} \mathrm{ha}^{-1}\right)$ of fine fuels (size $<6 \mathrm{~mm}$ ), larger fuels (size 6-75 mm) and duff loads in both the Italian fuel survey (red) and in the Fine Fuel Classification System database (blue).

cover (standardized loading $=0.83)$ and cli- $\quad$ (Fig. 4). Fine dead fuel loadings responded mate indices (thermotype loading $=0.77$, to increasing slope, southness and temperombrotype $=-0.68$ ), while axis 2 was ex- ature, while live grass and shrub loadings plained by a combination of all predictors increased in wetter ombrotypes and de-

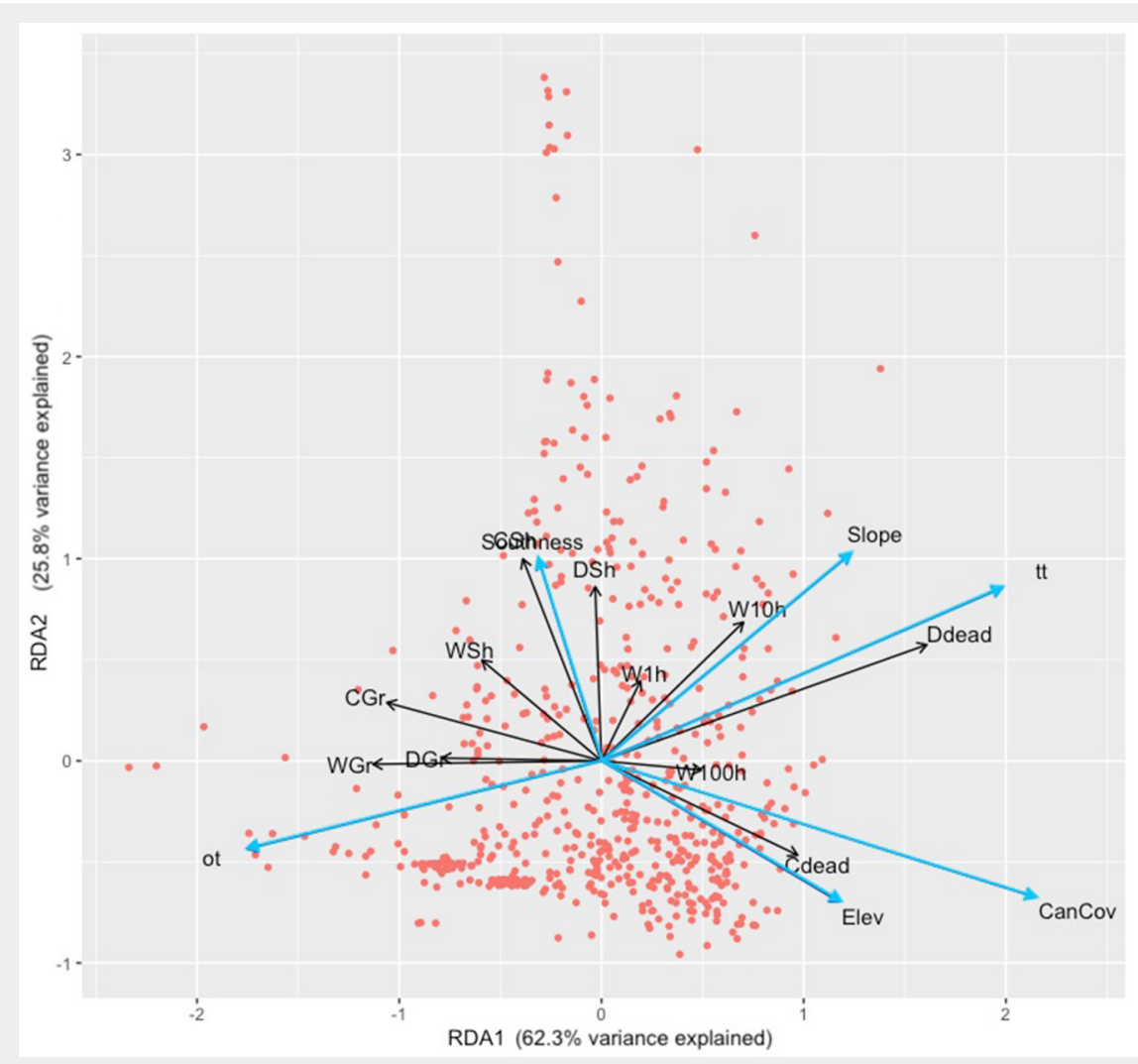

Fig. 4 - Biplot relationships between fuel load and site predictors. Red dots: individual sites; black arrow: response variables (W1h: fine dead fuel with particle size $<6 \mathrm{~mm}$; W10h: fine dead fuel with particle size 6-25 mm; W10oh: fine dead fuel with particle size $25-75 \mathrm{~mm}$; WGr: grass and grass live fuel < $6 \mathrm{~mm}$; WSh: shrub live fuel $<25 \mathrm{~mm}$ ); blue arrows: predictors (CanCov: canopy cover; Elev: elevation; Southness: -cos [aspect]; tt: thermotype in the bioclimate map of Italy according to Pesaresi et al. 2017, where increasing values correspond to decreasing temperatures; ot: ombrotype in the bioclimate map of Italy according to Pesaresi et al. 2017, where increasing values correspond to increasing aridity). Predictors were scaled to improve graphical representation. 
creasing elevation and canopy cover. The latter was true also for live grass depth and cover; dead fuel depth and cover were more strongly associated to thermotype/ slope and canopy cover/elevation, respectively, while live shrub depth and cover responded mostly to increasing southness (Fig. 4).

\section{Discussion}

The fuel dataset deriving from this study provides quantitative characteristics of fuel beds in grasslands, shrublands, broadleaved and coniferous forests of the Alpine, temperate and Mediterranean regions of Italy. It includes information useful for estimates on fuel biomass, surface fire behavior and effects. Although the sample size was limited and unevenly spatially distributed across the Italian peninsula, the survey covered a broad range of bioclimatic regions and vegetation types. This is apparent from the associations established between the 19 fuelbeds and the European Forest Types (Barbati et al. 2014), and the forest categories of the Italian National Forest Inventory (INFC 2005). Although resulting from an a posteriori compilation of fuel observations from different surveys, the fuel dataset covers all the classes of forest, grassland and shrubland mapped in Italy by Corine Land Cover IV level (ISPRA 2010).

In this Italian fuel dataset, the fuelbed with the lowest total surface fuel load was "Sparse and very short grasslands" with an average of $2.47 \mathrm{Mg} \mathrm{ha}^{-1}$. This was due to the dominance of very short grasses with a relatively low cover, which is typical of nutrient-poor and climatically limited sites such as those at high elevation or on very dry sites (fuelbed 1, Fig. S1 in Supplementary material). On the contrary, the fuelbed with the highest total surface fuel load (duff excluded) is "Aquatic marshes". These are very flammable fuel complexes with high spatial continuity, a deep fuelbed (326 $\pm 19 \mathrm{~cm}$ on average), and dead fuels $<25 \mathrm{~mm}$ representing $40 \%$ of total surface fuel load (fuelbed 19, Fig. S1 in Supplementary material).

Fuel characteristics of Italian vegetation fuels are within the range of values in the Fuel Characteristics Classification System (FCCS), one of the most extensive databases on fuel load and structure (Ottmar et al. 2007). Only fine fuel loads ( $<6 \mathrm{~mm})$ were markedly lower than FCCS loads. This could be related to the higher variability of vegetation types included in FCCS (from tropical to boreal ecosystems), specific fuelbeds with very high biomass levels such as "White fir-giant sequoia-sugar pine forest", and an older stage of development of North American forests (Cheyette et al. 2008), leading to higher productivity when compared to Italian forests. Fuel inventories carried out in similar fuelbeds displayed comparable fuel loads for both Alpine and Mediterranean shrublands. For example, Fréjaville et al. (2018) reported a total fuel load (excluding duff) of 7.7-12.3 $\mathrm{Mg}$ ha $^{-1}$ in Alpine mountainous forest categories, which falls within the range of 6.2 to $17.2 \mathrm{Mg} \mathrm{ha}^{-1}$ for fuelbeds 7 to 15 (Tab. 3), i.e., mountain forests under Alpine and temperate conditions. For Mediterranean shrublands, Dimitrakopoulos (2002) reported a total fuel load in Kermes oak and tall evergreen sclerophylous shrubland (excluding duff and diameters $>7.5 \mathrm{~cm}$ ) of 34.7 and $46.9 \mathrm{Mg} \mathrm{ha}^{-1}$, respectively, which falls within the range of fuelbed 6 "Tall Mediterranean shrublands and heathlands" of $16.8-53 \cdot 3 \mathrm{Mg}$ ha $^{-1}$ (Tab. 2). Similarly, fuel load in Mediterranean grasslands according to Dimitrakopoulos (2002) was $4.85 \mathrm{Mg} \mathrm{ha}{ }^{-1}$, which approximates the mean total fuel load of $4.08 \mathrm{Mg} \mathrm{ha}^{-1}$ of fuelbed 2 "Continuous short grasslands". Total fuel load in long-needled Mediterranean pine forests with sparse understory in Fernandes (2009) ranged from 5.25 to 14.09 $\mathrm{Mg} \mathrm{ha}{ }^{-1}$, which is within the range of fuelbed 16 "Mediterranean long needled conifer litter" of 0.8-19.7 Mg ha-1. Furthermore, fuel loads in forest fuelbeds were similar to understory dry weight biomass values published by the Italian National Forest Inventory (INFC 2005). Indeed, mean litter biomass in the INFC (i.e., leaves and twigs up to $25 \mathrm{~mm}$ ) is $6.4 \mathrm{Mg} \mathrm{ha}^{-1}$ while in this fuel dataset the sum of $W 1 \mathrm{~h}$ and W1oh loads of forest fuelbeds (mean of fuelbeds 7 to 18 ) is $5.6 \pm 0.21 \mathrm{Mg} \mathrm{ha}{ }^{-1}$. Similarly, load of W10oh downed woody material $(25-75 \mathrm{~mm})$ was $2.1 \pm 0.19 \mathrm{Mg} \mathrm{ha}^{-1}$, which is close to INFC mean values for fine deadwood (25-94 mm), i.e., $1.8 \mathrm{Mg} \mathrm{ha}^{-1}$.

\section{Conclusions}

This study developed the first Italian vegetation fuel dataset based on 634 surface fuel complex observations collected within 337 study sites and covering the major types of ecosystems found in Italy. Overall, 19 fuelbeds were established, characterized by a set of fuel variables ( 41 fields), ranging from fuel component loads to the fuel type cover and depth. Although lacking the a priori sampling design needed for robust statistical inference, the fuelbed dataset presented in this study includes a broad set of fuel features that can be used for several modeling applications and decision support systems. For example, the range of fuel loadings can be used to calibrate Rothermel's fire behavior fuel models by genetic algorithms (Ascoli et al. 2015) against observed rate of spread in a given fire environment to achieve realistic fire simulations by Rothermel's based software and DSS (Vacchiano \& Ascoli 2015). The available fuelbed data can be also used to estimate fuel consumption and emissions of greenhouse gases and particulate through the application of tools such as FOFEM (Reinhardt et al. 1997) or CONSUME (Prichard 2007). Moreover, the fuel dataset provides ranges of carbon pools not yet available in the Italian National Forest Inventory such as grass and shrub biomass, despite their relevance for carbon inventories (Didion 2020). Further analysis of this data could allow to calibrate allometry relationships between above ground and dead biomass pools (Federici et al. 2008) to broad the carbon stock estimates in forest inventories. Additional field surveys and national sampling efforts, along with a standardization of the sampling scheme/approach, are certainly needed to enhance reliability and accuracy of estimates in fuelbeds/fuel complexes currently less represented (i.e., fuelbed 12 - Eucaliptus litter; fuelbed 18 - Riparian vegetation) or affected by high variability due to the intrinsic heterogeneity of the vegetation and site conditions. Future research will also foresee the association of the fuelbed characteristics to specific non spatial (e.g., forest typologies) and spatial (e.g., forest types maps) products commonly used in natural resource management at the regional scale in Italy. This approach will provide cost-effective fuel related information and maps providing many advantages for land and resource management analysis, e.g., fuel hazard assessment, preventive fire management measures, strategic fire management planning, through the prioritization of areas and resource allocation for fuel treatment to mitigate potential fire danger, risk, and fire emission and model carbon-cycle and air quality. Finally, spatially-explicit information coupled with future climate (Caddeo et al. 2019) and land use change scenarios (Martellozzo et al. 2018) would offer a valuable support towards more comprehensive landscape planning able to minimize risk related disturbances such as wildfires (Marchetti et al. 2014), as well as maximize ecosystem services provisioning and their trade-offs at the landscape level (Vizzarri et al. 2017).

\section{Acknowledgments}

The study is the result of a research program carried out by the "Fire Management Working Group" of the Italian Society of Silviculture and Forest Ecology (https:// sisef.org/category/gdl/incendi-boschivi).

\section{References}

Alvarez A, Gracia M, Vayreda J, Retana J (2012). Patterns of fuel types and crown fire potential in Pinus halepensis forests in the Western Mediterranean Basin. Forest Ecology and Management 270: 282-290. - doi: 10.1016/j.foreco.2011. 01.039

Andrews PL (2018). The Rothermel surface fire spread model and associated developments: a comprehensive explanation. Gen. Tech. Rep. RMRS-GTR-371, USDA Forest Service, Rocky Mountain Research Station, Fort Collins, CO, USA, pp. 121. [online] URL: http://www.fs.usda. gov/treesearch/pubs/55928

Andrews PL, Bevins CD, Seli RC (2005). BehavePlus fire modeling system, version 3.0: user's guide. Gen. Tech. Rep. RMRS-GTR-106, USDA Forest Service, Rocky Mountain Research Station, Ogden, UT, USA, pp. 132. - doi: 10.2737/ RMRS-GTR-106 
Arca B, Duce P, Laconi M, Pellizzaro G, Salis M, Spano D (2007). Evaluation of FARSITE simulator in Mediterranean maquis. International Journal of Wildland Fire 16: 563-572. - doi: 10.1071/WF06070

Arca B, Ghisu T, Casula M, Salis M, Duce P (2019). A web-based wildfire simulator for operational applications. International Journal of Wildland Fire 28: 99-112. - doi: 10.1071/WF18078

Ascoli D, Bovio G (2013). Prescribed burning in Italy: issues, advances and challenges. iForest Biogeosciences and Forestry 6 (2): 79-89. - doi 10.3832/iforo803-006

Ascoli D, Vacchiano G, Motta R, Bovio G (2015). Building Rothermel fire behaviour fuel models by genetic algorithm optimisation. Internation al Journal of Wildland Fire 24: 317-328. - doi: 10.1071/WF14097

Bacciu V, Arca B, Pellizzaro G, Salis M, Ventura A, Spano D, Duce $P$ (2009). Mediterranean maquis fuel model development and mapping to sup port fire modeling. In: "EGU General Assembly Conference Abstracts”. Vienna (Austria) 19-24 Apr 2009. Geophysical Research, pp. 13148. [on line] URL: http://ui.adsabs.harvard.edu/abs/200 9EGUGA..1113148B/abstract

Bacciu V, Scarpa C, Ascoli D, Salis M, Sirca C, Marchetti M, Spano D (2018). Extreme fire events in summer 2017 accounted for a large portion of carbon loss and national greenhouse gas emissions in Southern Italy. In: Proceedings of the "IV National Congress of Silviculture". Torino (Italy) 5-9 Nov 2018. Accademia Italiana di Scienze Forestali, Firenze, Italy, pp. 312. [in Italian]

Barbati A, Marchetti M, Chirici G, Corona P (2014). European forest types and forest Europe SFM indicators: tools for monitoring progress on forest biodiversity conservation. Forest Ecology and Management 321: 145-157. doi: 10.1016/j.foreco.2013.07.004

Bebi P, Kulakowski D, Veblen TT (2003). Interac tions between fire and spruce beetles in a sub alpine Rocky Mountain forest landscape. Ecology 84 (2): 362-371. - doi: 10.1890/0012-9658(20 03)084[0362:IBFASB]2.0.CO;2

Beck HE, Zimmermann NE, McVica TR, Vergopolan N, Berg A, Wood EF (2018). Present and fu ture Köppen-Geiger climate classification maps at 1-km resolution. Scientific Data 5: 180-214. doi: 10.1038/sdata.2018.214

Bovio G, Ascoli D (2013). La tecnica del fuoco prescritto [Prescribed burning technique]. AR ACNE editrice srl, Roma, Italy, pp. 272. [in Italian]

Bovio G, Corona P, Leone V (2014). Gestione selvicolturale dei combustibili forestali per la prevenzione degli incendi boschivi [Silvicultural management of forest fuels for the prevention of forest fires] (vol. 2). Compagnia delle For este, Arezzo, Italy, pp. 208. [in Italian]

Brown JK, Oberheu R, Johnston C (1982). Handbook for inventory surface fuels and biomass in the interior West. Gen. Tech. Rep. INT-129, USDA Forest Service, Intermountain Forest and Range Experiment Station, Ogden, UT, USA, pp. 48.

Caddeo A, Marras S, Sallustio L, Spano D, Sirca C (2019). Soil organic carbon in Italian forests and agroecosystems: estimating current stock and future changes with a spatial modelling ap proach. Agricultural and Forest Meteorology 278: 107654. - doi: 10.1016/j.agrformet.2019.107 654

Castagneri D, Esposito A, Bovio G, Mazzoleni S, Seneca U, Catalanotti AE, Ascoli D (2013). Fuel vertical structure affects fire sustainability and behaviour of prescribed burning in Spartium junceum shrublands. Annals of Forest Science 70: 863-871. - doi: 10.1007/s13595-013-0327-3

Cheyette D, Rupp ST, Rodman S (2008). Developing fire behavior fuel models for the wildland-urban interface in Anchorage, Alaska. West Journal of Applied Forestry 23: 149-155. doi: $10.1093 /$ wjaf/23.3.149

Conti G, Gorné LD, Zeballos SR, Lipoma ML, Gatica G, Kowaljow E, Whitworth-Hulse JI, Cuchietti A, Poca M, Pestoni S, Fernandes PM (2019). Developing allometric models to predict the individual aboveground biomass of shrubs worldwide. Global Ecology and Biogeography 28: 961-975. - doi: 10.1111/geb.12907

Corona P, Pasta S, Giardina G, La Mantia T (2012). Assessing the biomass of shrubs typical of Mediterranean pre-forest communities. Plant Biosystems 146: 252-257. - doi: 10.1080/11263504. 2011.593200

Corona P, Ascoli D, Barbati A, Bovio G, Colangelo G, Elia M, Garfì V, lovino F, Lafortezza R, Leone V, Lovreglio R, Marchetti M, Marchi E, Menguzzato G, Nocentini S, Picchio R, Portoghesi L, Puletti N, Sanesi G, Chianucci F (2015). Integrated forest management to prevent wildfires under Mediterranean environments. Annals of Silvicultural Research 39: 1-22. - doi: 10.12899/ASR946

Cruz MG, Gould JS, Hollis JJ, McCaw W (2018). A hierarchical classification of wildland fire fuels for Australian vegetation types. Fire 1 (1): 13 . doi: $10.3390 /$ fire1010013

Didion M (2020). Extending harmonized national forest inventory herb layer vegetation cover observations to derive comprehensive biomass estimates. Forest Ecosystems 7: 1-14. - doi: 10.11 86/s40663-020-00230-7

Dimitrakopoulos AP (2002). Mediterranean fuel models and potential fire behaviour in Greece. International Journal of Wildland Fire 11 (2): 127130. - doi: 10.1071/WF02018

Dimitrakopoulos AP, Mitsopoulos ID, Gatoulas K (2010). Assessing ignition probability and moisture of extinction in a Mediterranean grass fuel. International Journal of Wildland Fire 19 (1): 29-34. - doi: 10.1071/WFo8124

Duce P, Pellizzaro G, Arca B, Ventura A, Bacciu V, Salis M, Bortolu S, Spano D, Santoni PA, Barboni T, Leroy V, Cancellieri D, Leoni E, Ferrat L, Perez $Y$ (2012). Fuel types and potential fire behavior in Sardinia and Corsica islands: a pilot study. In: "Modelling Fire Behaviour and Risk" (Spano D, Bacciu V, Salis M, Sirca C eds). Nuova StampaColor Muros, Italy, pp. 208.

EFFIS (2017). European fuel map, 2017. JRC Contract no. 384347 "Development of a European Fuel Map”, European Forest Fire Information System - EFFIS, European Commission, Bruxelles, Belgium. [online] URL: https://effis.jrc.ec. europa.eu/applications/data-and-services/

Elia M, Lafortezza R, Lovreglio R, Sanesi G (2015). Developing custom fire behavior fuel models for Mediterranean wildland-urban interfaces in southern Italy. Environmental man- agement 56: 754-764. - doi: 10.1007/s00267-0150531-z

Elia M, Lovreglio R, Ranieri N, Sanesi G, Lafortezza R (2016). Cost-effectiveness of fuel removals in Mediterranean wildland-urban interfaces threatened by wildfires. Forests 7 (12): 149. - doi: 10.3390/f7070149

Elia M, D’Este M, Ascoli D, Giannico V, Spano G, Ganga A, Colangelo G, Lafortezza R, Sanesi C (2020). Estimating the probability of wildfire occurrence in Mediterranean landscapes using Artificial Neural Networks. Environmental Impact Assessment Review 85: 106474. - doi: 10.1016/j.eiar.2020.106474

Federici S, Vitullo M, Tulipano S, De Lauretis R, Seufert G (2008). An approach to estimate carbon stocks change in forest carbon pools under the UNFCCC: the Italian case. iForest - Biogeosciences and Forestry 1: 86-95. - doi: 10.3832/ iforo457-0010086

Fernandes PM (2009). Combining forest structure data and fuel modelling to classify fire hazard in Portugal. Annals of Forest Science 66 (4): 1-9. - doi: 10.1051/forest/2009013

Finney MA (1998). FARSITE: fire area simulatormodel development and evaluation. Research Paper RMRS-RP-4, USDA Forest Service, Rocky Mountain Research Station, Ogden, UT, USA, pp. 47. [online] URL: http://books.google.com/ books?id=WdAXbjQz12YC

Finney MA (2006). An overview of FlamMap fire modeling capabilities. In: Proceedings of the "Fuels management - How to measure success" (Andrews PL, Butler BW eds). Portland (OR, USA) 28-30 Mar 2006. USDA Forest Service, Rocky Mountain Research Station, Fort Collins, CO, USA, pp. 213-220. [online] URL: http://www.fs.usda.gov/treesearch/pubs/25948 Fréjaville T, Curt T, Carcaillet C (2018). Higher potential fire intensity at the dry range margins of European mountain trees. Journal of Biogeography 45: 2003-2015. - doi: 10.1111/jbi.13386

Incerti G, Giordano D, Stinca A, Senatore M, Termolino P, Mazzoleni S, Bonanomi G (2013). Fire occurrence and tussock size modulate facilitation by Ampelodesmos mauritanicus. Acta oecologica 49: 116-124. - doi: 10.1016/j.actao.2013.03. 012

INFC (2005). Inventario nazionale delle foreste e dei serbatoi forestali di carbonio [National inventory of forests and forest carbon stocks]. Ministero delle Politiche Agricole Alimentari e Forestali, Ispettorato Generale - Corpo Forestale dello Stato - CRA, Unità di ricerca per il Monitoraggio e la Pianificazione forestale, Trento, Italy. [in Italian]

ISPRA (2010). La realizzazione in Italia del progetto Corine Land Cover 2006 [The realization in Italy of the project Corine Land Cover 2006]. Rapporti 131/2010, ISPRA, pp. 50. [in Italian]

Keane RE (2013). Describing wildland surface fuel loading for fire management: a review of approaches, methods and systems. International Journal of Wildland Fire 22: 51-62. - doi: 10.1071/WF11139

Keane RE (2015). Wildland fuel fundamentals and applications. Springer International Publishing, Cham, Switzerland, pp. 191. - doi: 10.1007/978-3-319-09015-3

Marchetti M, Vizzarri M, Lasserre B, Sallustio L, Tavone A (2014). Natural capital and bioecon- 
omy: challenges and opportunities for forestry. Annals of Silvicultural Research 38: 62-73. - doi: 10.12899/asr-1013

Maringer J, Wohlgemuth T, Hacket-Pain A, Ascoli D, Berretti R, Conedera M (2020). Drivers of persistent post-fire recruitment in European beech forests. Science of the Total Environment 699: 134006. - doi: 10.1016/j.scitotenv.201 9.134006

Martellozzo F, Amato F, Murgante B, Clarke KC (2018). Modelling the impact of urban growth on agriculture and natural land in Italy to 2030. Applied Geography 91: 156-167. - doi: 10.1016/j. apgeog.2017.12.004

Ottmar RD, Sandberg DV, Riccardi CL, Prichard SJ (2007). An overview of the fuel characteristic classification system quantifying, classifying, and creating fuelbeds for resource planning. Canadian Journal of Forest Research 37: 23832393. - doi: 10.1139/X07-077

Ottmar RD (2014). Wildland fire emissions, carbon, and climate: modeling fuel consumption. Forest Ecology and Management 317: 41-50. doi: 10.1016/j.foreco.2013.06.010

Pellizzaro G, Cesaraccio C, Duce P, Ventura A, Zara P (2007). Relationships between seasonal patterns of live fuel moisture and meteorological drought indices for Mediterranean shrubland species. International Journal of Wildland Fire 16 (2): 232-241. - doi: 10.1071/WF06081

Pesaresi S, Biondi E, Casavecchia S (2017). Bioclimates of Italy. Journal of Maps 13: 955-960. doi: $10.1080 / 17445647.2017 .1413017$

Prichard SJ (2007). Consume 3.0 user's guide. USDA Forest Service, Web site. [online] URL: http://www.fs.fed.us/pnw/fera/research/smoke /consume/

R Core Team (2019). R: a language and environment for statistical computing. R Foundation for Statistical Computing, Vienna, Austria. [online] URL: http://www.r-project.org/

Reinhardt ED, Keane RE, Brown JK (1997). First order fire effects model: FOFEM 4.0, User's
Guide. General Technical Report INT-GTR-344, USDA Forest Service, Intermountain Forest and Range Experiment Station, Ogden, UT, USA, pp. 65. [online] URL: http://books.google.com/ books?id=3HngDt6 6TYC

Rivas-Martínez S, Sáenz SR, Penas A (2011). Worldwide bioclimatic classification system. Global Geobotany 1: 1-634.

Rothermel RC (1972). A mathematical model for predicting fire spread in wildland fuels. Research Paper INT-115, USDA Forest Service, Intermountain Forest and Range Experiment Station, Ogden, UT, USA, pp. 40. [online] URL: http://books.google.com/books?id=AfyMv5NBS joC

Salis M, Ager AA, Arca B, Finney MA, Bacciu V, Duce P, Spano D (2013). Assessing exposure of human and ecological values to wildfire in Sardinia, Italy. International Journal of Wildland Fire 22: 549-565. - doi: 10.1071/WF11060

Salis M, Laconi M, Ager AA, Alcasena FJ, Arca B, Lozano OM, Oliveira AS, Spano D (2016). Evaluating alternative fuel treatment strategies to reduce wildfire losses in a Mediterranean area. Forest Ecology and Management 368: 207-221. doi: 10.1016/j.foreco.2016.03.009

Sandberg DV, Ottmar RD, Cushon GH (2001). Characterizing fuels in the $21^{\text {st }}$ century. International Journal of Wildland Fire 10: 381-387. - doi: 10.1071/WF01036

Sirca C, Caddeo A, Spano D, Bacciu V, Marras S (2016). Methods for biomass stock estimation in Mediterranean maquis systems. iForest - Biogeosciences and Forestry 10 (1): 108-114. - doi: 10.3832/ifor1769-009

Ubysz B, Valette JC (2010). Flammability: influence of fuel on fire initiation. In: "Towards Integrated Fire Management - Outcomes of the European Project Fire Paradox" (Sande Silva J, Rego F, Fernandes P, Rigolot E eds). Research Report 23, European Forest Institute - EFI, Joensuu, Fl, pp. 23-34.

Vacchiano G, Motta R, Bovio G, Ascoli D (2014).
Calibrating and testing the forest vegetation simulator to simulate tree encroachment and control measures for heathland restoration in Southern Europe. Forest Science 60: 241-252. doi: 10.5849/forsci.12-064

Vacchiano G, Ascoli D (2015). An implementation of the Rothermel fire spread model in the $R$ programming language. Fire Technology 51: 523-535. - doi: 10.1007/s10694-014-0405-6

Vizzarri M, Sallustio L, Travaglini D, Bottalico F, Chirici G, Garfì V, Lafortezza R, Salvatore D, Veca LM, Lombardi F, Maetzke F, Marchetti M (2017). The MIMOSE approach to support sustainable forest management planning at regional scale in Mediterranean contexts. Sustainability 9 (2): 316. - doi: 10.3390/sug020316

\section{Supplementary Material}

Tab. S1 - Fields of the Italian fuel characteristics dataset.

Tab. S2 - Foliage and branches fuel load and source of data by fuel complex.

Fig. S1 - Scheme showing the structure of the dataset as regards quantitative variables characterizing each fuel observation.

Fig. S2 - Photos describing the 19 fuelbeds included in the dataset.

\section{Link: Ascoli_3587@supploo1.pdf}

Tab. S3 - Harmonized fuel characteristics dataset including 634 fuel samples and 41 variables (see Tab. S1) at 377 sites under Alpine, Temperate and Mediterranean conditions in Italy.

Link: Ascoli_3587@supploo2.xlsx 